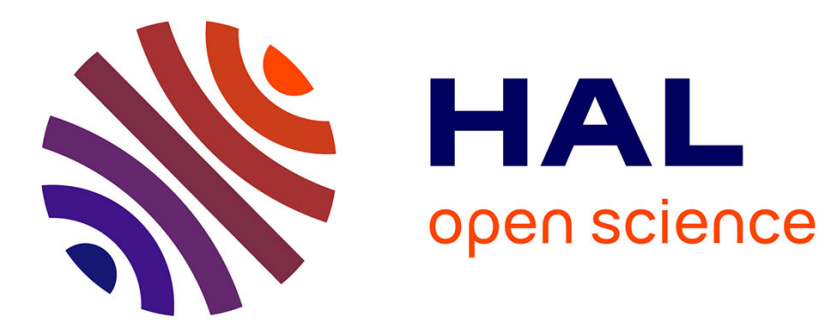

\title{
Wood Furniture Waste-Based Recycled 3-D Printing Filament
}

\author{
Adam M Pringle, Mark Rudnicki, Joshua Pearce
}

\section{To cite this version:}

Adam M Pringle, Mark Rudnicki, Joshua Pearce. Wood Furniture Waste-Based Recycled 3-D Printing

Filament. Forest products journal, 2017, 68, 10.13073/FPJ-D-17-00042 . hal-02111355

\section{HAL Id: hal-02111355 \\ https://hal.science/hal-02111355}

Submitted on 26 Apr 2019

HAL is a multi-disciplinary open access archive for the deposit and dissemination of scientific research documents, whether they are published or not. The documents may come from teaching and research institutions in France or abroad, or from public or private research centers.
L'archive ouverte pluridisciplinaire HAL, est destinée au dépôt et à la diffusion de documents scientifiques de niveau recherche, publiés ou non, émanant des établissements d'enseignement et de recherche français ou étrangers, des laboratoires publics ou privés. 
Preprint: Adam M. Pringle, Mark Rudnicki, and Joshua M. Pearce.Wood Furniture Waste-Based Recycled 3-D Printing Filament. Forest Products Journal 2018, Vol. 68, No. 1, pp. 86-95. https://doi.org/10.13073/FPJ-D-17-00042

\title{
Wood Furniture Waste-Based Recycled 3-D Printing Filament
}

\author{
Adam M. Pringle, Mark Rudnicki, and Joshua Pearce
}

\begin{abstract}
The Michigan furniture industry produces $>150$ tons/day of wood-based waste, which can be upcycled into a wood polymer composite (WPC). This study investigates the viability of using furniture waste as a feedstock for 3-D printer filament to produce furniture components. The process involves: grinding/milling board scraps made of both LDF/MDF/LDF and melamine/particleboard/paper impregnated with phenolic resins; pre-mixing wood-based powder with the biopolymer poly lactic acid (PLA), extruding twice through open-source recyclebots to fabricate homogeneous 3-D printable WPC filament, and printing with open source FFF-based 3-D printers. The results indicate there is a significant opportunity for waste-based composite WPCs to be used as 3-D printing filament.
\end{abstract}

\section{Introduction}

The extraordinary number of uses for wood demonstrates its value and versatility, which also extends to its ability to be reused and recycled. In addition, wood is a renewable resource that sequesters and stores carbon, making wood increasingly viewed as the material of choice for a future not based on petrochemicals (Jakes, 2016). Extending the life of wood while increasing its value through upcycling, presents opportunities for innovation that brings us closer to a sustainable future.

For example, the Michigan furniture industry produces more than 150 tons of wood-based waste per day (Gurn, 2016), which is primarily landfilled and burned in a 38MW wood waste to energy plant in Flint, MI (Michigan Biomass, 2017). However, this waste has the potential to be upcycled into wood plastic composites (WPCs) rather than burned, as there are well-documented challenges for waste to energy plants (Pavlas et al., 2010; Tabasová, et al., 2012). WPCs are composites containing a wood component in particle form but within a polymer matrix that can provide greater performance, reduced price, and reduced environmental impact (Schwarzkopf, 2016). There is a wide range of modification techniques for wood either involving active modifications such as thermal or chemical treatments, or passive modification, which changes the physical properties but not the biochemical structure (Ormondroyd, 2015). However, WPCs still have limitations due to production methods, such as producing waste material or orientation reliant fabrication, which may be alleviated with alternative manufacturing techniques such as additive manufacturing.

Additive manufacturing (or 3-D printing) is different from subtractive manufacturing processes, which typically involve grinding, cutting, or milling, because 3-D printers function by building a part layer-by-layer, each as thin as 50 microns. This allows for a significant reduction in scrap and wasted material as well as a unique mentality when designing parts meant specifically for this manufacturing method (Steenhui, 2015). Though 3-D printing was developed several decades ago, significant growth has occurred within the last decade as 3-D printing has become more accessible to consumers (Wohlers, 2014). The release of the open source RepRap (self REPlicating RAPid prototyper) 3-D printers has radically decreased the costs of 3-D printers (Sells et al., 2009; Jones et al., 2011; Bowyer, 2014; Rundle, 2014). RepRaps use fused filament fabrication (FFF) where a thin polymer extrusion from a heated print head is layered, where it cools and connects to surrounding polymer (Steenhui, 2015). Many polymers are available on the market for FFF 3-D printing including nylon, polycarbonate (PC), high-density polyethylene (HDPE), and high impact polystyrene (HIPS), etc., (Hunt et al., 2015). However, the most common FFF material is polylactic acid (PLA), which has an average tensile strength of 56.6 MPa (Tymrak et al., 2014), is relatively inexpensive, has a glass transition temperature of $45-60^{\circ} \mathrm{C}$ and melting point of $150-178^{\circ} \mathrm{C}$ (Farah, 2016), making it suitable for a wide range of applications, especially for 3-D printing. PLA is a bio-based plastic made up a repeating chain of lactic acid and it is re cyclable using conventional methods. Specifically, PLA is a thermoplastic aliphatic polyester derived from corn and can even be composted like other organic matter. The low glass transition temperature and high melting temperature create a large temperature region allowing for semi-solid material flow. This is ideal for additive depositions as it in creases the printability of the material. Printability being the ease at which a material is to work with and produce desired parts from a digital design. PLA is versatile and PLA-based composites have been investigated within the litera- 
Preprint: Adam M. Pringle, Mark Rudnicki, and Joshua M. Pearce.Wood Furniture Waste-Based Recycled 3-D Printing Filament. Forest Products Journal 2018, Vol. 68, No. 1, pp. 86-95. https://doi.org/10.13073/FPJ-D-17-00042

ture including composites with natural fibers (Oksman, et al., 2003), kenaft fibers (Ochi, 2003), microfibrillated cellulose (Suryanegara, et al., 2009), man-made cellulose and abaca fibers (Bledzki, et al., 2009), carbon nanotube (Kuan, et al., 2008) and even metal composites (Fortunati, et al., 2012; Laureto, 2017). There are already several PLA composite manufacturers in the market including Laywood, Gizmo Dorks, and SainSmart (All3DP, 2017; Gizmo Dorks, 2009; SainSmart, 2014), which create virgin wood-based 3-D printer filament.

This study furthers these efforts by investigating the use of furniture waste as a feedstock for 3-D printer WPC filament to produce currently purchased furniture components. The process uses grinding and milling of two furniture waste materials - boards scraps made of both LDF/MDF/LDF (where LDF is light density fill and MDF is medium density fill) and melamine/particleboard/paper impregnated with phenolic resins. A pre-mixing process is used for the resultant wood-based powder with PLA pellets (NatureWorks, 4043D). This material is extruded twice through an open source recyclebot to fabricate homogeneous 3-D printable filament in volume fractions of wood:PLA from 10:100 to 40:100. The filament is tested in an open source FFF-based industrial 3-D printer. The results are presented and discussed to analyze the opportunity for waste based composite filament production.

\section{Methodology}

In this work, four steps have been developed for the production of WPCs from furniture wood waste for 3-D printing filament. Wood-based waste material was received from several furniture manufacturing companies in both bulk (half meter size slabs) as well as sawdust (mm to sub-mm size). The first step in the method is size reduction from macro-scale and meso-scale to the micro-scale. Once the furniture wood-based waste is fine enough in particle size (about 80microns), the wood-based filler material with the consistency of baking flour is mixed with the matrix polymer. The third step is extruding the feed material into filament of homogeneous thickness and density to provide uniform properties. The last step is to load the WPC filament into a 3-D printer and print a part. To print a part an object must be modeled in a 3-D modeling software, imported into a slicing software, and then uploaded to a 3-D printer's firmware.

\subsection{Furniture Wood-Based Waste Material Particle Size Reduction}

The material was received in a few different sizes and were LDF/MDF/LDF and melamine/particleboard/paper impregnated with phenolic resins. These materials were used as obtained and not cleaned in order to more thoroughly test the recyclability of wood waste material. Whatever surface contaminates were on the as received materials, would likely be negligible due to the sheer amount of new surfaces generated when particles are reduced to micron size. Larger materials were broken up initially via band saw into 10x6 cm sections for feeding into a wood chipper (seen in Figure 1A). The material was cycled through the wood chipper three times and the different sources of wood-based furniture waste were mixed together to form an even composition weight percent (wt\%) of each supplier's materials. This combined furniture waste mix had a nominal particle size of 2-3mm wide and 2-6 mm in length. At this size they were too large to be added to the PLA polymer for 3-D printing through a standard nozzle ( $0.5 \mathrm{~mm}$ in diameter), but could used on larger nozzles, to achieve a particleboard type print. As the goal of this work is to investigate fine structures, the material was hammer milled (Figure 1B) with various mesh sizes ranging from $3.00 \mathrm{~mm}$ to $0.75 \mathrm{~mm}$ in in batches of $200 \mathrm{~g}$ (50g from each supplier) for 30 minutes; at each mesh resulting a sub-mm average size of wood fiber mix. Initial trials of filament that were fabricated with wood fibers of this size met with reasonable success, however the particle size was determined to be too large for a consistent FFF extrusion through a 0.5mm nozzle on a standard delta type RepRap 3-D printer (Irwin, et al., 2014; Anzalone, et al., 2015). Therefore, a fine particle sifter assembly (Pringle, 2017) was fabricated (Figure 1C) and another assembly was printed out of PLA on a 3-D printer. The sifter assembly is made up of four sections, the bucket for particle collection, the replaceable stainless steel mesh (Kindustrial), the funnel for guiding particle flow, and the lid for sealing to prevent loss of fine particles. One assembly incorporated a 210-micron stainless steel mesh and the other an 80-micron stainless steel mesh. The sifting was automated using a vibratory de-airing device (model H-1756 from Humboldt) to produce sub 80-micron particles. Material was first loaded into the 210-micron mesh sifter and held onto the vibratory device with rubber bands to allow optimal shaking. The material initially vibrated for one hour before being transferred to the 80-micron mesh sifter and vibrated again for one hour. By the end of the process, the powder 
Preprint: Adam M. Pringle, Mark Rudnicki, and Joshua M. Pearce.Wood Furniture Waste-Based Recycled 3-D Printing Filament. Forest Products Journal 2018, Vol. 68, No. 1, pp. 86-95. https://doi.org/10.13073/FPJ-D-17-00042

material had the consistency of grain flour and will now be referred to as wood-waste powder. Throughout this process the moisture content was never directly measured. Through observation of the wood-waste powder material lack of agglomeration and ease of free-flow when handled, it was concluded that the heat generated during the size reduction process reduced moisture content to acceptable levels.

\subsection{Filler-matrix mixing}

The temperature of PLA pellets (NatureWorks, 4043D) was raised to $210^{\circ} \mathrm{C}$ until the PLA became less viscous and could be stirred. The wood-waste powder was added gradually and both materials were stirred and mechanically mixed (Figure 2A). The mix was allowed to regain $210^{\circ} \mathrm{C}$ temperature to compensate for the temperature drop upon material addition and reduce viscosity. Batch sizes above $20 \mathrm{wt} \%$ wood-waste or larger than 100 grams the woodwaste powder did not completely wet into the PLA matrix due to the thermal gradient created from the directional heat flow from the heating element. Therefore, longer stir times were conducted as batch sizes increased to improve wettability. Additionally, stir speed was increased to prevent the wood powder from charring at the mixing temperature. After a consistent mixed material was obtained, the batch was removed from the heating element and allowed to cool to room temperature exposed to air and placed on a metal surface to act as a heat sink (Figure 2B) before it was fed into a wood chipper to obtain a particle size of roughly 3-4 $\mathrm{mm}$ in diameter with a non-uniform angular geometry (Figure 2C).

\subsection{Filament Extrusion}

The chipped WPC material (Figure 2C) was then further homogenized by being added to an open-sourced recyclebot (Baechler, et al., 2013). The recyclebot is a waste plastic extruder that fabricates filament for FFF 3-D printers. The recyclebot used in this study was a vertical design (Figure 3B) and a detailed procedure on its creation is described by Zhong et al. (Zhong et al., 2017; Zhong and Pearce, 2018). The motor is capable of 15 rpm when set at max, but only should be done so during the homogenization step, with the end nozzle off to prevent mechanical backlash to the motor. Wound nichrome wire $15 \mathrm{~cm}$ in length down the exterior of the barrel tube allows a uniform maximum temperature of $225^{\circ} \mathrm{C}$. Due to the non-uniform geometries the angular WPC material as received from the wood chipper, feeding into the recyclebot was difficult, however, it was reformed into a cylindrically shaped prefilament material (Figure 2D). This pre-filament was unsuitable for printing as the diameter varied significantly, as an average was $1.45 \mathrm{~mm}$ in diameter $(+/-0.3 \mathrm{~mm})$ because of inconsistent feeding of material (volumetric flow) into the auger. The pre-filament was then processed in the wood chipper again to obtain uniform cylindrical pellets. The now cylindrical WPC pellets allowed consistent volumetric flow into the recyclebot auger (primarily due to the rolling edge) allowing spooling of the material with more uniform diameter $(+/-0.1 \mathrm{~mm})$. Before filament fabrication the auger of the recyclebot was purged of old material to prevent contamination by addition of pure PLA pellets (Figure 3A), which were run through the recyclebot until clear PLA filament was being extruded. Then, the cylindrical WPC pellets were loaded into the designed and printed recyclebot hopper (Pringle, 2017) for a final run through the recyclebot to extrude WPC filament. During the extrusion process the aircooled WPC filament was then positioned through the photodiode at the bottom of the recyclebot, fed through several sensors (diameter and length) and attached to the auto-spooler. Correct thickness of filament is critical to have sufficient latent heat maintaining a flexible core, but allowing a smooth and non-sticky exterior to the filament. The photodiode measures how far the filament produced dips and adjusts the spooling speed to maintain a constant gravitational pool of the filament exiting the extruder to maintain a consistent diameter (Figure 3B). The degree of WPC filament thinning of the filament is adjustable by changing the distance between the extrusion hole and the photodiode sensor. Filaments of wood weight percentages 10, 20, 30 and $40 \mathrm{wt} \%$ wood were produced. The observable rheology of the WPC filament changed in small, but noticeable part due to increasing wood content. First, the higher the wood content the more opaque and wood-like the filament appeared. There was a slight increase in the rigidity of the polymer as wood content increased, and was determined through observations of handling the filament in spooled form and how easily each filament composition unspooled. 
Preprint: Adam M. Pringle, Mark Rudnicki, and Joshua M. Pearce.Wood Furniture Waste-Based Recycled 3-D Printing Filament. Forest Products Journal 2018, Vol. 68, No. 1, pp. 86-95. https://doi.org/10.13073/FPJ-D-17-00042

\subsection{3-D Printing WPC Process}

The final step in the methodology is 3-D printing a desired part with the WPC filament created using a delta RepRap and an open source Re:3D Gigabot version GB2 (re:3D, 2013). An open-source tool chain was used in all aspects of 3-D printing shown in Figure 4A-C. First, OpenSCAD version 2015.03-2 (OpenSCAD, 2010), a scriptbased 3-D modeling program was used for part design. After a part is modeled it is exported as an STL file (stereolithography) and imported into a slicer program, Cura version 2.3.1 (Ultimaker, 2012) to be exported as gcode. Cura allowed precise control of relevant printing parameters such as temperature, layer height and printing speed summarized in Table 1. The gcode is sent to Franklin (Wijnen, et al., 2016), which is an open source firmware and control software. Franklin allows direct and real-time control of temperature, extrusion speed, cooling rate, as well as motor speed. Screenshots of OpenSCAD, Cura, and Franklin are displayed in Figure 4.

The spooled filament diameter was measured by a caliper to ensure it was sufficient consistency ( $\pm 0.10 \mathrm{~mm})$. In order to feed properly into a $0.5 \mathrm{~mm}$ hot end, a diameter of less than $2 \mathrm{~mm}$ is necessary to prevent binding of the filament. Several part files including a desktop cable feed through and various desk and drawer knobs as well as multiple blocks were fabricated using the values in Table 1 and primarily 30wt\% filament. While pure PLA is printed normally at 210 ${ }^{\circ} \mathrm{C}$, a temperature of $185^{\circ} \mathrm{C}$ was used in order to reduce the amount of charring the wood component of the filament would undergo through printing. As material is printed, the heat flow and buildup must be taken into account. Decreasing the print speed, increasing the print temperature, or decreasing the feed rate (mass flow rate) all increase the exposure of heat for a given section of filament. Likewise, if the print speed increases, the print temperature increases, or the mass flow rate increases, any given section of the print will be overall cooler as the total heat energy is dissipated over a larger volume.

Table 1: 3-D Printing parameters used for recycled wood-furniture waste based filament.

\begin{tabular}{|c|c|}
\hline Printing Parameter & Value \\
\hline Layer height & $0.15 \mathrm{~mm}$ \\
\hline Shell thickness & $1 \mathrm{~mm}$ \\
\hline Top/bottom thickness & $1 \mathrm{~mm}$ \\
\hline Fill density & $33 \%$ \\
\hline Printing temperature & $185^{\circ} \mathrm{C}$ \\
\hline Filament diameter & $1.65 \mathrm{~mm}$ \\
\hline Nozzle size & $0.5 \mathrm{~mm}$ \\
\hline Print speed & $62.5 \mathrm{~mm} / \mathrm{s}$ \\
\hline Bottom layer speed & $25 \mathrm{~mm} / \mathrm{s}$ \\
\hline Travel speed & $100 \mathrm{~mm} / \mathrm{s}$ \\
\hline
\end{tabular}


Preprint: Adam M. Pringle, Mark Rudnicki, and Joshua M. Pearce.Wood Furniture Waste-Based Recycled 3-D Printing Filament. Forest Products Journal 2018, Vol. 68, No. 1, pp. 86-95. https://doi.org/10.13073/FPJ-D-17-00042

\section{Results and Discussion}

\subsection{Recycled Wood Furniture Waste-based WPC Filament and Repeatability}

Uniform and homogeneous recycled wood furniture waste-based WPC filament was produced with the above described methodology and is shown in Figure 5A. Filament was measured to have diameter consistency of $1.65 \pm 0.10 \mathrm{~mm}$. This is not ideal, as most commercial filament is $1.75 \pm 0.05 \mathrm{~mm}$ in tolerance. However, the extruder drive on the delta 3-D printer was be adjusted through modifying the tightness of the idler against the filament to account for the difference in filament diameter. A 3-D printed desk cable feedthrough (shown in Figure 5B) was used to test the viability of producing a purchased part for furniture manufacturing, test filament printing parameters and demonstrate repeatability. The simple part has a flat base with a cylindrical wall and hollow center. Additionally, the part had a hole near the top and it was important to maintain a circular shape. The weight percent that demonstrated the most success and ease of creation was $30 \mathrm{wt} \%$. This wt $\%$ was chosen based primarily on wettability of wood-waste powder into PLA as well as printability of the resulting filaments.

It is also important to note that during the filament extrusion and printing processes, there were no material flow disruptions attributed to excess moisture content. These disruptions would be characterized by small "pop" sounds coming from the nozzle as polymeric material high in moisture are extruded as the water vapor is released. High moisture content would lead to porosity and inconsistent filament diameter. This effect can sometimes be witnessed in normal printing settings if filament is left in an environment with high humidity levels due to the hygroscopic nature of some 3-D printing filaments. Unfortunately, both wood and PLA are hygroscopic and composite filament of these two materials should be kept in a controlled low-humidity environment.

The molecular weight of a polymer determines the physical properties. As polymers are exposed to elevated temperatures, they may degrade. Rheological studies of PLA from temperatures of 180 to $200^{\circ} \mathrm{C}$ has been completed in the literature (Al-Itry, et al., 2012). In their work, viscosity of PLA changed from $2800 \mathrm{~Pa}$ s to $1250 \mathrm{~Pa}$ s when processed at $180^{\circ} \mathrm{C}$ and $200^{\circ} \mathrm{C}$, respectively. Decrease in viscosity corresponds to a reduction in molecular weight. While exposed to these temperatures in the short term, increased chain mobility occurs, extended influence leads to thermal degradation and thus reduction in molecular weight as polymer chains breakdown. For recycled materials, this means there is a lifetime limit for the number of times a given batch of PLA may be recycled into filament and printed before mechanical properties degrade enough to prevent usage (Cruz et al., 2016).

Phenolic resins or melamine resins have negligible impact on the extrusion of the filament produced with this methodology. These resins are thermosetting polymers and will decompose before transitioning to a liquid state, meaning they may be treated as solid particulates. This research has found that resins, if they do not decompose in the filament fabrication process, due to elevated temperatures, behave similarly to wood particles. Resin gets broken down in the reduction steps as does wood. The particle size of the resins, in general, then matches the wood particle sizes. While this means, resins may be treated as wood in terms of particle size, the material properties of the filament can change to reflect the properties of the resin. Filaments with higher concentrations of resin may show increased mechanical properties, water resistance, and heat resistance. In this work, resin if not broken down to similar particle size, would have been sifted out early in the process.

\subsection{3-D Printing Techniques for Recycled Wood Furniture Waste-based WPC Filament}

The recycled wood furniture waste-based WPC filament produced parts without many errors once an optimized printing profile was obtained. However, when compared to traditional filament such as pure PLA, there were still a greater frequency of nozzle clogging and general filament blockages, most likely caused by particle size non-uniformity. When comparing the compositions, higher wood-waste percentages did demonstrate more clogging, but this is likely due to having more oversized particles for the selected nozzle size. Blockages occur when the nozzle of the hot end has restricted flow. Contamination on, within the filament, or the ratio of the particle size versus the nozzle head diameter may restrict flow and cause a clog to occur. Contamination such as dust may settle on filament when left unattended in a printing environment. To prevent this contamination, a sponge was taped around the filament prior to entry into the printer to collect all surface contamination. Contamination within the filament would 
Preprint: Adam M. Pringle, Mark Rudnicki, and Joshua M. Pearce.Wood Furniture Waste-Based Recycled 3-D Printing Filament. Forest Products Journal 2018, Vol. 68, No. 1, pp. 86-95. https://doi.org/10.13073/FPJ-D-17-00042

come from either the wood-waste material or PLA pellets harboring unintended materials such as oils, but the concentrations necessary would need to be substantial and would likely prevent filament from even forming into shape. The smaller the wood particles with a larger nozzle would reduce clogging potential, however, a smaller nozzle allows higher levels of precision in the 3-D printed part. A Gigabot by Re3D was also tested for capability with the wood filament produced and no blockages were observed during the duration of the experiment. If nozzle size is appropriate for the particle size, the rate of clogging should be no different than for pure PLA filaments. Figure 6A-C shows several parts mid print with both the Gigabot and delta RepRap.

Varying the print temperature, print speed or extrusion rate changes the volumetric flow rate of material through the hot region of the hot end assembly on a FFF-based 3-D printer. As material passes through the hot region the wood particles begin to char at a rate depending on how much time it takes a given volume of material to pass through and out of the nozzle. Longer duration will lead to darker colorations in the printed part. When blockages occurs, if not all of the material is removed then the remaining material will blacken and may lead to additional clogging issues. It is also important to note that with larger nozzle diameters, higher temperatures are necessary to uniformly heat the increased volumetric flow allowed for reliable inner-layer bonding. By varying one of the three parameters mentioned above, the layers of a print may be colored to resemble the wood grain of traditional products (Kaipa, 2012), this is shown in Figure 7B. By far the easiest parameter to change is the print speed as it may be done instantaneously in the Franklin firmware during printing. The print temperature is also simple to change, however, it is a delayed response as the hot end must then warm or cool to the new temperature. Furthermore, if the print speed or print temperature, or extrusion rate is adjusted too much in one direction (more or less) then print quality may suffer if the filament material does not have sufficient time in the hot region to adequately transition towards the necessary material viscosity for printing.

Because 3-D printing allows for precise control of printing parameters the internal geometry of printed parts it is not constrained to traditional manufacturing requirements. Thus, an ideal internal geometry may be selected,many are available in open source slicing programs such as Slic3r, for the intended application (Fedorov, et al., 2012). Figure 7A shows a $20 \%$ infill triangular geometry for a drawer handle. Printing with lower infills has already been shown to be a key method to reduce the environmental impact of 3-D printing compared to conventional products (Kreiger and Pearce, 2013a;b) as well as reduce the material used, mass and cost of the part. However, it is always important to note the layer deposition direction and if possible print a part to experience forces perpendicular to the layer direction so the force acts on the layers in total of the affected region, rather than on the inner-layer adhesion of the printed part (Vega et al., 2011).

\subsection{Example Application of Recycled Wood Furniture Waste-based WPC Filament}

WPCs have many varied applications, which are expanded with the use of 3-D printing. To demonstrate this a highresolution drawer knob was 3-D printed attached to a printed wood block using a wood screw threaded through a preprinted hole as shown in Figure 8A-B. The wood screw was easily twisted through both objects with a Phillips screwdriver and the resulting connection withstood normal forces expected in everyday use. Additionally due to the flexibility of 3-D printing orientations a unique or personalized surfaces may be printed onto objects as shown in Figure 9. This is shown through the particular geometries or print directions which may be modified directly by altering gcode, or more conveniently by changing parameters in slicer programs. This enables mass-scale personalization of not only furniture components with wood, but any 3D printed part using recycled waste-based plastic composites

As the weight percent of wood furniture waste filler increases in a given batch of material, the properties of the 3-D printed parts more closely resemble that of wood. With parts printed of at least $15 \mathrm{wt} \%$ wood furniture waste, the parts began to take on the smell and texture of wood with higher weights exhibiting a stronger scent. In addition, 3-D printed parts could also be sanded, stained, and painted like normal wood products, which further increases the value of these WPC products. Furthermore, throughout this project it became apparent that wasted wood parts or failed prints were also able to be recycled and the material reused. This was done, by feeding the discarded parts into a wood chipper and the methodology repeated to obtain filament once again. This recycling of printed parts did not seem 
Preprint: Adam M. Pringle, Mark Rudnicki, and Joshua M. Pearce.Wood Furniture Waste-Based Recycled 3-D Printing Filament. Forest Products Journal 2018, Vol. 68, No. 1, pp. 86-95. https://doi.org/10.13073/FPJ-D-17-00042

overly detrimental as long as temperatures did not exceed $210^{\circ} \mathrm{C}$ for long durations, which would have led to degradation.

\section{Future Work}

Future work is needed to quantify the mechanical properties of the furniture waste WPC after the first cycle and compare it to pure PLA, modified wood fiber powder WPCs (Zhang et al., 2016) and other and other wood fiber biocomposites (Le Duigou et al., 2016). As polymeric materials are recycled, they tend to degrade, and this is shown in the reduction in molecular weight. Work should be done on measuring the impact on the mechanical properties of polymeric materials with different molecular weights resulting from degradation during the recycling process. Improvement of the wettability of the wood-waste powder is essential to gain higher weight percentages in 3-D printing filaments. Other polymer materials should also be tested and compared to assess the ideal matrix for wood composite waste, the matrix likely has a large impact on wettability of the aggregate material. In addition, the impact of multiple cycles through the recyclebot should be tested (Cruz, et al., 2016) and with the addition of colorants, which have been shown to have an effect (Wittbrodt and Pearce, 2015). The scale-up of this process should also be evaluated with industrial equipment, larger sized and grouped or ganged 3-D print nozzles (multiple nozzles per print bed to make several parts at once) would improve output production. A continuous process of the four steps mentioned to streamline the filament production would be necessary for industry volume requirements.. In addition, the environmental and economic performance of furniture waste WPCs should be evaluated. Finally, the market for sale of furniture wood based WPC should be evaluated in the context of an ethical filament market (Feeley, et al., 2014).

\section{Conclusions}

This study has demonstrated a technically viable methodology of upcycling furniture wood waste into usable 3-D printable parts for the furniture industry. By mixing PLA pellets and recycled wood waste material filament was produced with a diameter size of $1.65 \pm 0.10 \mathrm{~mm}$ and used to print a small variety of test parts. This method while developed in the lab may be scaled up to meet industry needs as the process steps are uncomplicated. Small batches of $40 \mathrm{wt} \%$ wood were created, but showed reduced repeatability, while batches of 30wt\% wood showed the most promise with ease of use.

\section{Acknowledgements}

This research was made possible by a gift to the Michigan Tech Fund. Contributing organizations include Gunlocke, Inc., Haworth, Inc., Herman Miller, Inc., Jasper Group \& Brands, Steelcase, Inc., Trendway Corporation, and BIFMA, Inc.

\section{Literature Cited}

3D Slicer. Available at: https://www.slicer.org/. Accessed: 17th February 2017

All3DP. 2017. 30 Types of 3D Printer Filament - Guide \& Comparison Chart. Available at: https://all3dp.com/best-3dprinter-filament-types-pla-abs-pet-exotic-wood-metal/ Accessed: 17th February 2017

Al-Itry, R., Lamnawar, K. \& Maazouz, A. 2012. Improvement of thermal stability, rheological and mechanical properties of PLA, PBAT and their blends by reactive extrusion with functionalized epoxy. Polymer Degradation and Stability $97(10)$, 1898-1914

Anzalone, G.C., Wijnen, B. \& Pearce, J.M. 2015. Multi-material additive and subtractive prosumer digitalfabrication with a free and open-source convertible delta RepRap 3-D printer. Rapid Prototyp. J. 21(5):506-519.

Baechler, C., DeVuono, M. and Pearce, J.M., 2013. Distributed recycling of waste polymer into RepRap feedstock. Rapid Prototyping Journal, 19(2), pp.118-125. 
Preprint: Adam M. Pringle, Mark Rudnicki, and Joshua M. Pearce.Wood Furniture Waste-Based Recycled 3-D Printing Filament. Forest Products Journal 2018, Vol. 68, No. 1, pp. 86-95. https://doi.org/10.13073/FPJ-D-17-00042

Bledzki, A.K., Jaszkiewicz, A. and Scherzer, D., 2009. Mechanical properties of PLA composites with man-made cellulose and abaca fibres. Composites Part A: Applied science and manufacturing, 40(4):404-412.

Bowyer, A. 2014. 3D printing and humanity's first imperfect replicator, 3D printing and additive manufacturing, Vol. $1(1): 4-5$.

Cruz, F., Lanza, S., Boudaoud, H., Hoppe, S. and Camargo, M., 2016. Polymer Recycling and Additive Manufacturing in an Open Source context: Optimization of processes and methods. Annual International Solid Freeform Fabrication Symposium - An Additive Manufacturing Conference 1591-1600. https://sffsymposium.engr.utexas.edu/sites/default/files/2015/2015-127-Cruz.pdf

Ultimaker. 2012. Cura 3D Printing Slicing Software Ultimaker.com Available at: https://ultimaker.com/en/products/cura-software. Accessed: 17th February 2017

Farah, S., Anderson, D.G., Langer, R., 2016. Physical and mechanical properties of PLA, and their functions in widespread applications - A comprehensive review. Advanced Drug Delivery Reviews, PLA biodegradable polymers 107, 367-392.

Fedorov A., Beichel R., Kalpathy-Cramer J., Finet J., Fillion-Robin J-C., Pujol S., Bauer C., Jennings D., Fennessy F., Sonka M., Buatti J., Aylward S.R., Miller J.V., Pieper S., Kikinis R. 2012. 3D Slicer as an Image Computing Platform for the Quantitative Imaging Network. Magnetic Resonance Imaging. 30(9):1323-41. PMID: 22770690.

Feeley, S.R., Wijnen, B. and Pearce, J.M., 2014. Evaluation of potential fair trade standards for an ethical 3-D printing filament. Journal of Sustainable Development, 7(5), 1.

Fortunati, E., Armentano, I., Zhou, Q., Puglia, D., Terenzi, A., Berglund, L.A. and Kenny, J.M., 2012. Microstructure and nonisothermal cold crystallization of PLA composites based on silver nanoparticles and nanocrystalline cellulose. Polymer degradation and stability, 97(10):2027-2036.

Gizmo Dorks. 2009. 3D Printer Wood Filament. Available at: http://gizmodorks.com/wood-3d-printer-filament/. Accessed: 17th February 2017.

Gurn, Bill. March 2016. Personal communication. Manager of Haworth production facility. Holland Michigan.

Hunt, E.J., Zhang, C., Anzalone, N. and Pearce, J.M., 2015. Polymer recycling codes for distributed manufacturing with 3-D printers. Resources, Conservation and Recycling, 97:24-30.

Irwin, J.L., Pearce, J.M., Anzolone, G. \& Oppliger, D.E. 2014. The RepRap 3-D printer revolution in STEM education. In 121st ASEE Annual Conference \& Exposition. Indianapolis, IN, USA, June 15-18, 2014, Paper ID \#8696.

Jones, R., Haufe, P., Sells, E., Iravani, P., Olliver, V., Palmer, C. and Bowyer, A. 2011. RepRap - the replicating rapid prototype, Robotica, 29(1):177-191.

Kaipa. 2012. Print wood now - with tree rings! LAYWOO-D3 mm filament http://www.thingiverse.com/thing:30552

Kreiger, M. and Pearce, J.M., 2013. Environmental impacts of distributed manufacturing from 3-D printing of polymer components and products. In MRS Proceedings Vol. 1492:85-90 Cambridge University Press.

Kreiger, M. and Pearce, J.M., 2013. Environmental life cycle analysis of distributed three-dimensional printing and conventional manufacturing of polymer products. ACS Sustainable Chemistry \& Engineering, 1(12):1511-1519. 
Preprint: Adam M. Pringle, Mark Rudnicki, and Joshua M. Pearce.Wood Furniture Waste-Based Recycled 3-D Printing Filament. Forest Products Journal 2018, Vol. 68, No. 1, pp. 86-95. https://doi.org/10.13073/FPJ-D-17-00042

Kuan, C.F., Kuan, H.C., Ma, C.C.M. and Chen, C.H., 2008. Mechanical and electrical properties of multi-wall carbon nanotube/poly (lactic acid) composites. Journal of Physics and Chemistry of Solids, 69(5):1395-1398.

Laureto, J., Tomasi, J., King, J.A., Pearce, J.M. 2017. Thermal Properties of 3-D Printed Polylactic Acid - Metal Composites. Progress in Additive Manufacturing, 2(1-2), 57-71.

Le Duigou, A., Castro, M., Bevan, R. and Martin, N., 2016. 3D printing of wood fibre biocomposites: from mechanical to actuation functionality. Materials \& Design, 96:106-114.

Ochi, S., 2008. Mechanical properties of kenaf fibers and kenaf/PLA composites. Mechanics of materials, 40(4):446452.

Ormondroyd, G., Spear, M. and Curling, S., 2015. Modified wood: review of efficacy and service life testing. Proceedings of the Institution of Civil Engineers-Construction Materials, 168(4):187-203.

Oksman, K., Skrifvars, M. and Selin, J.F., 2003. Natural fibres as reinforcement in polylactic acid (PLA) composites. Composites science and technology, 63(9):1317-1324.

OpenSCAD. 2010. Available at: http://openscad.org. Accessed: 17th February 2017

Michigan Biomass. 2017. Genesee Power Station. http://www.michiganbiomass.com/gps.php Accessed February 9 , 2017.

Pavlas, M., Touš, M., Bébar, L. and Stehlík, P., 2010. Waste to energy-an evaluation of the environmental impact. Applied Thermal Engineering, 30(16):2326-2332.

Pringle A. 2017. Recycle Bot Feeder Redesign v02. YouMagine Available at: https://www.youmagine.com/designs/recycle-bot-feeder-redesign-v02. Accessed: 21st February 2017

Pringle A. 2017. Parametric Modular Sifter-Bucket-Funnel Assembly. YouMagine Available at: https://www.youmagine.com/designs/parametric-modular-sifter-bucket-funnel-assembly. Accessed: 21st February 2017

Re:3D. 2013.Life-Sized Affordable 3D Printing. Available at https://re3d.org/. Accessed: 17th February 2017

Rundle, G. 2014. “A Revolution in the Making”. Affirm Press; South Melbourne.

SainSmart. 2014. 3D Printer Wood Filament 1.75mm 1kg Light Brown 3D Printing, Arduino, Robotics | Sainsmart. Available at: http://www.sainsmart.com/sainsmart-3d-printer-wood-filament-1-75mm-1kg-light-brown.html. Accessed: 17th February 2017

Schwarzkopf, M.J. and Burnard, M.D., 2016. Wood-Plastic Composites-Performance and Environmental Impacts. In Environmental Impacts of Traditional and Innovative Forest-based Bioproducts pp. 19-43. Springer Singapore.

Sells, E., Smith, Z., Bailard, S., Bowyer, A. and Olliver, V. 2009. RepRap: The Replicating Rapid Prototyper: Maximizing Customizability by Breeding the Means of Production, In Piller, F. T., Tseng, M. M. (Eds.). Handbook of Research in Mass Customization and Personalization: Strategies and concepts. World Scientific Vol 1:568-580, 2010.

Steenhuis, H.J. and Pretorius, L., 2015. Additive manufacturing or 3D printing and its adoption. International Association for Management of Technology, 2468-2479. 
Preprint: Adam M. Pringle, Mark Rudnicki, and Joshua M. Pearce.Wood Furniture Waste-Based Recycled 3-D Printing Filament. Forest Products Journal 2018, Vol. 68, No. 1, pp. 86-95. https://doi.org/10.13073/FPJ-D-17-00042

Suryanegara, L., Nakagaito, A.N. and Yano, H., 2009. The effect of crystallization of PLA on the thermal and mechanical properties of microfibrillated cellulose-reinforced PLA composites. Composites Science and Technology, 69(7):1187-1192.

Tabasová, A., Kropáč, J., Kermes, V., Nemet, A. and Stehlík, P., 2012. Waste-to-energy technologies: Impact on environment. Energy, 44(1):146-155.

Tymrak, B. M., Kreiger, M. and Pearce, J. M. 2014. Mechanical properties of components fabricated with open-source 3-D printers under realistic environmental conditions, Materials \& Design, Vol. 58, 242-246.

Vega, V., Clements, J., Lam, T., Abad, A., Fritz, B., Ula, N. and Es-Said, O.S., 2011. The effect of layer orientation on the mechanical properties and microstructure of a polymer. Journal of materials engineering and performance, 20(6):978-988.

Wijnen, B., Anzalone, G.C., Haselhuhn, A.S., Sanders, P.G. and Pearce, J.M., 2016. Free and open-source control software for 3-D motion and processing. Journal of Open Research Software, 4(1). http://openresearchsoftware.metajnl.com/articles/10.5334/jors.78/

Wittbrodt, B. and Pearce, J.M., 2015. The effects of PLA color on material properties of 3-D printed components. Additive Manufacturing, 8, pp.110-116.

Wohlers, T. and Caffrey, T. 2014. Wohlers Report 2014 Annual Worldwide Progress Report. Wohlers Associates, Inc. Fort Collins, CO.

Zhang, J.S., Yang, Y.T., Qin, Z.K., Luo, J.J., Gao, W. and Wei, S.L., 2016. Research Progress of the Modified Wood Powder for 3D printing. http://www.atlantis-press.com/php/download paper.php?id=25859966

Zhong, S., Rakhe, P., Pearce, J.M. 2017. Energy Payback Time of Solar Photovoltaic Powered Waste Plastic Recyclebot System. Recycling, 2(2), 10; doi:10.3390/recycling2020010

Zhong, S., Pearce, J.M. 2018. Tightening the Loop on the Circular Economy: Coupled Distributed Recycling and Manufacturing with Recyclebot and RepRap 3-D Printing. Resources, Conservation and Recycling, 128, 48-58. 
Preprint: Adam M. Pringle, Mark Rudnicki, and Joshua M. Pearce.Wood Furniture Waste-Based Recycled 3-D Printing Filament. Forest Products Journal 2018, Vol. 68, No. 1, pp. 86-95. https://doi.org/10.13073/FPJ-D-17-00042

\section{Figures}
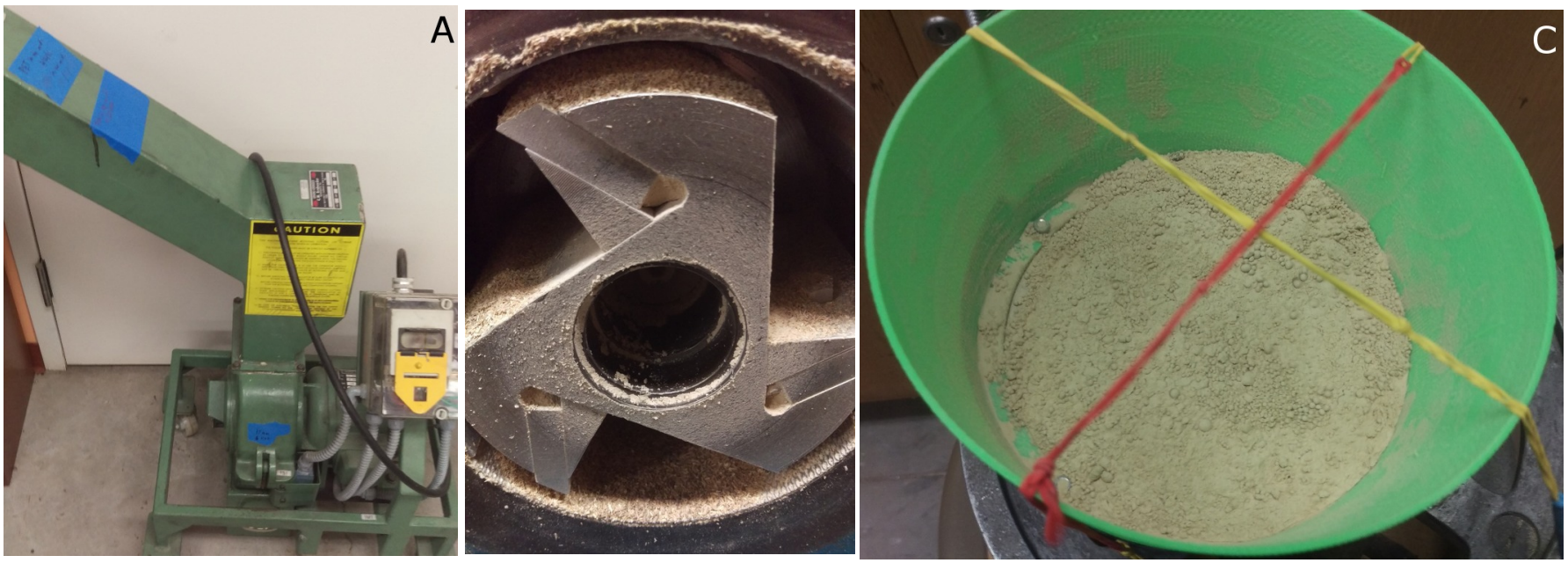

Figure 1.-A) Wood chipper, B) Hammer mill, and C) 80-micron sifter assembly for wood-particle size reduction.
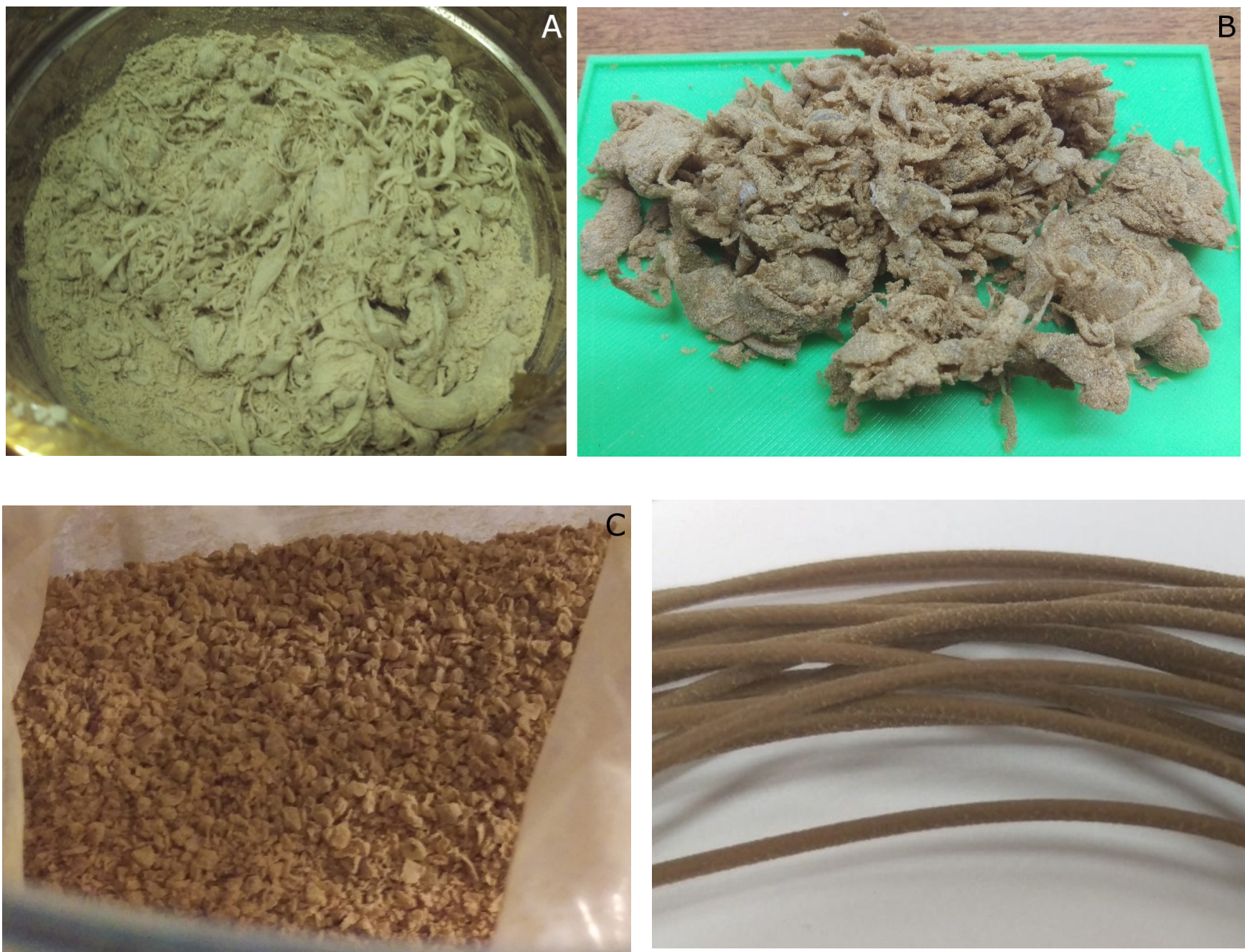

Figure 2. A) PLA during moderate mechanical mixing with wood-waste powder, B) PLA and wood-waste powderbased WPC after mixing and cooling to room temperature, C) chipped WPC, D) homogeneous WPC material after first pass through recyclebot. 

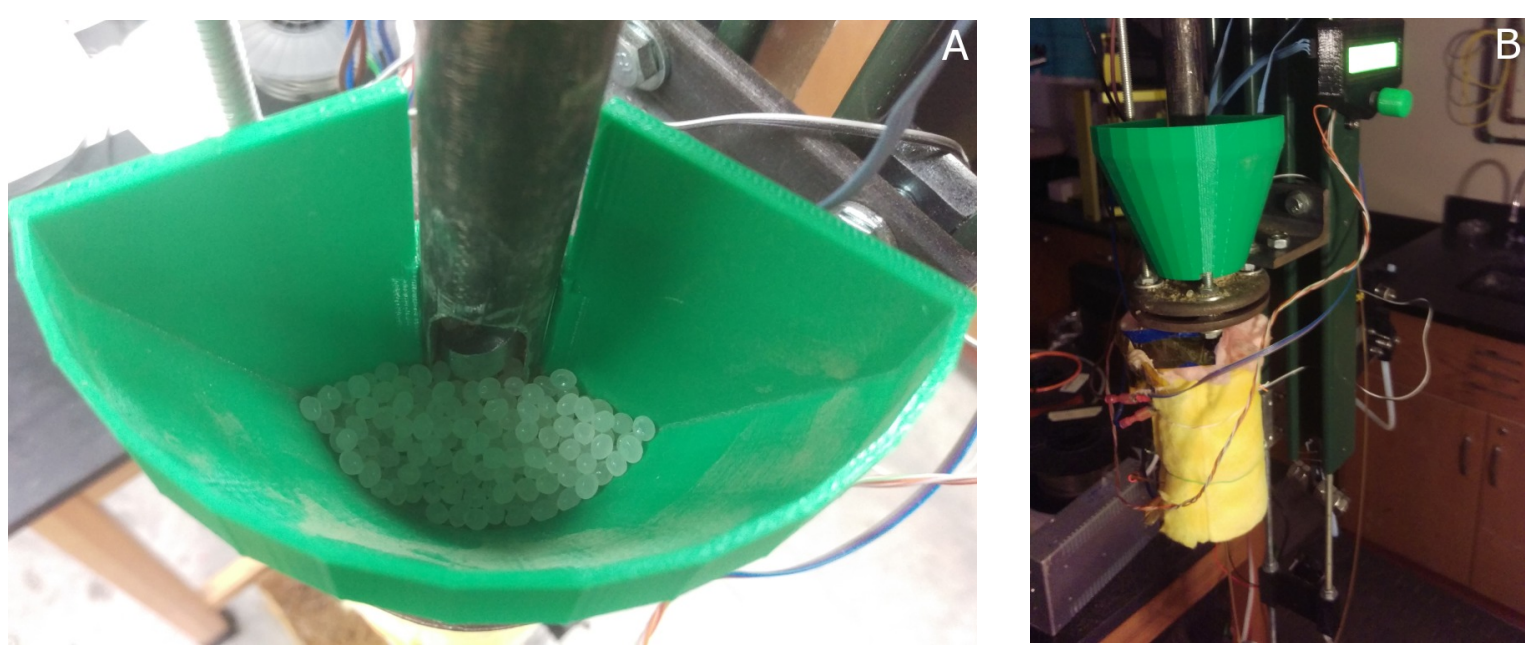

Figure 3: A) vertical recyclebot hopper with pure PLA pellets, B) vertical recyclebot showing hopper, heating zone, photodiodes (bottom), control and spooler (upper right).
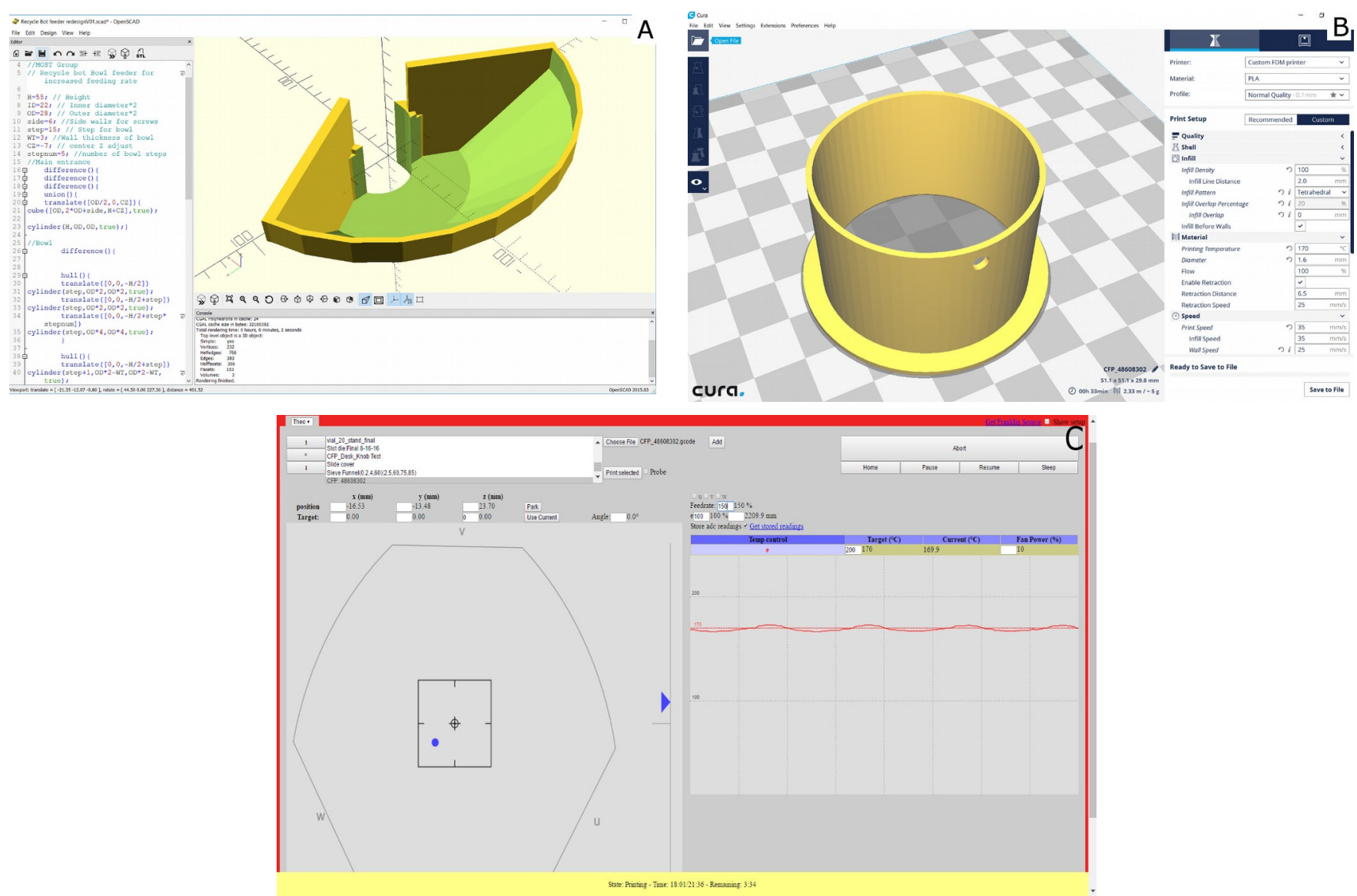

Figure 4: A) OpenSCAD, B) Cura, and C) Franklin are used in the open-source part creation process. 

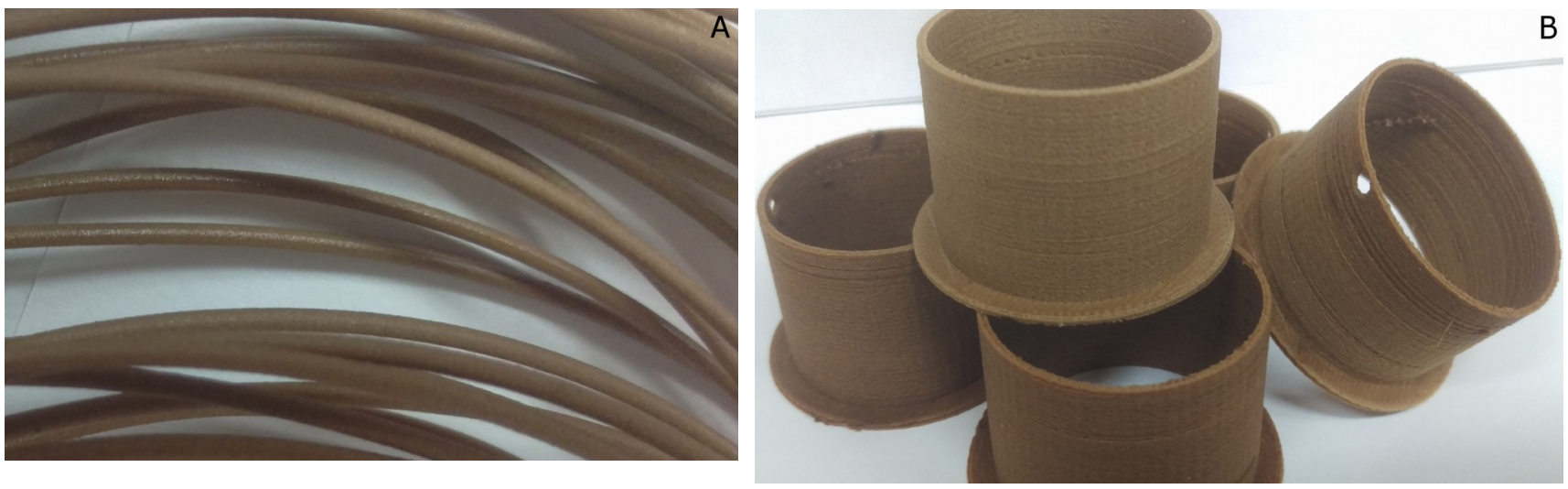

Figure 5: A) spooled recycled wood furniture waste-based WPC filament (removed from spool) with 30wt\% woodbased waste. B), five desk cable feedthrough parts 3-D printed consecutively, 30wt\% wood furniture-based waste.
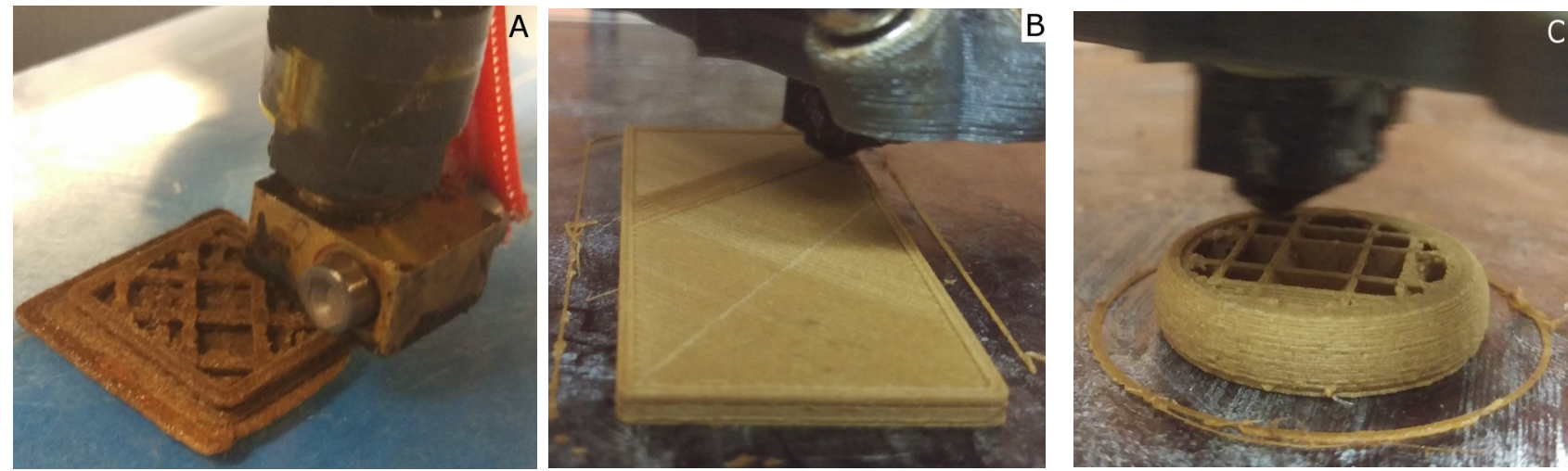

Figure 6: A) Test cube done on Gigabot with 20\% infill, 25wt\% wood furniture waste. B) Test block done on delta with $100 \%$ infill, $30 \mathrm{wt} \%$ wood furniture waste. C) Layer 34 of a drawer knob done on delta with tetrahedral shaped internal geometry, 30wt\% wood furniture waste.
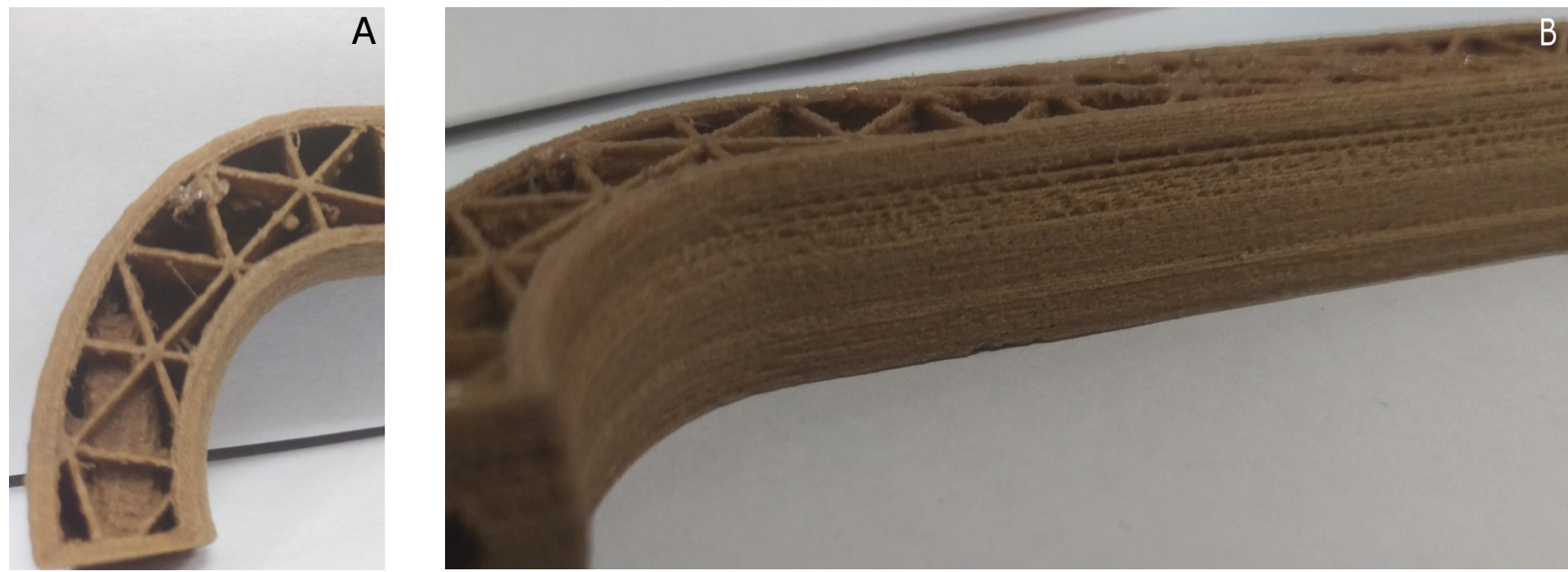

Figure 7: A) Internal view of a drawer handle with triangular geometry of infill, 30wt\% wood furniture waste. B) Side view of same drawer handle, sidewalls have been textured during print to resemble an aesthetic wooden surface. 


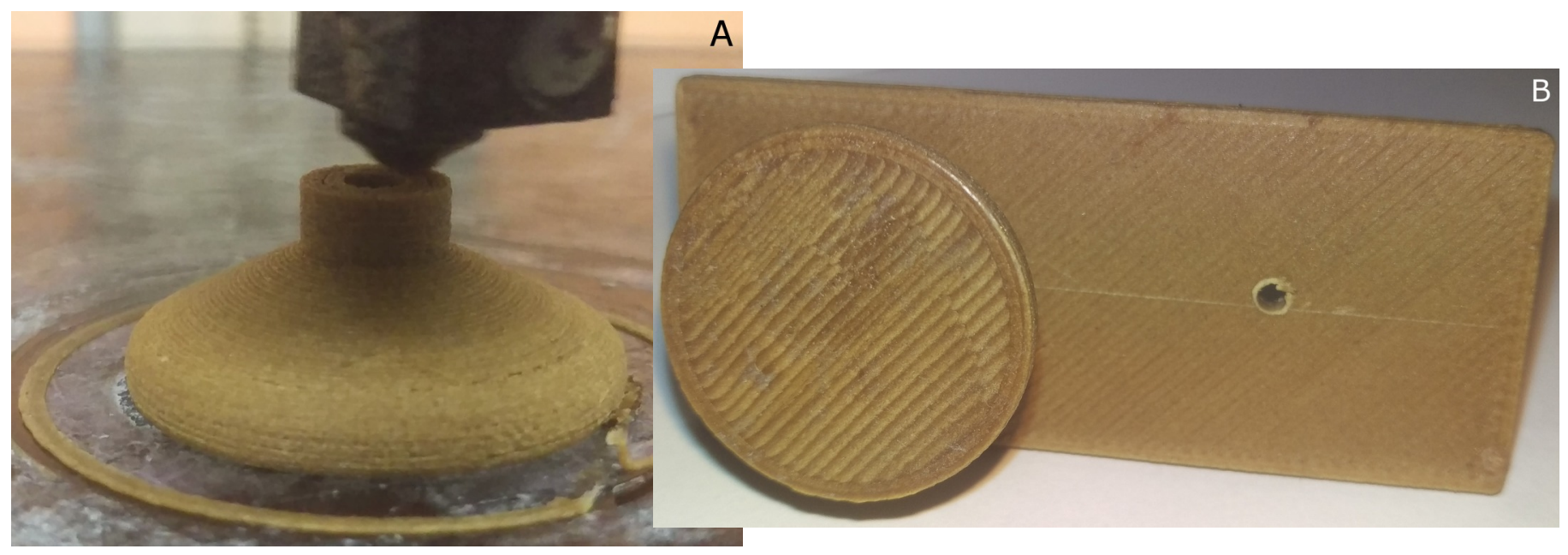

Figure 8: A) $0.15 \mathrm{~mm}$ layer height drawer knob being 3-D printed with a screw hole for attachment. B) Completed drawer knob fully attached on left of wood block with example pre-printed hole on right of block, 30wt\% wood furniture waste.

Figure 9: Surface contours of a personalized drawer handle for with the Herman Miller emblem. A coloration change from the outsides to the center is shown due to induced temperature changes during printing to provide a tree ring

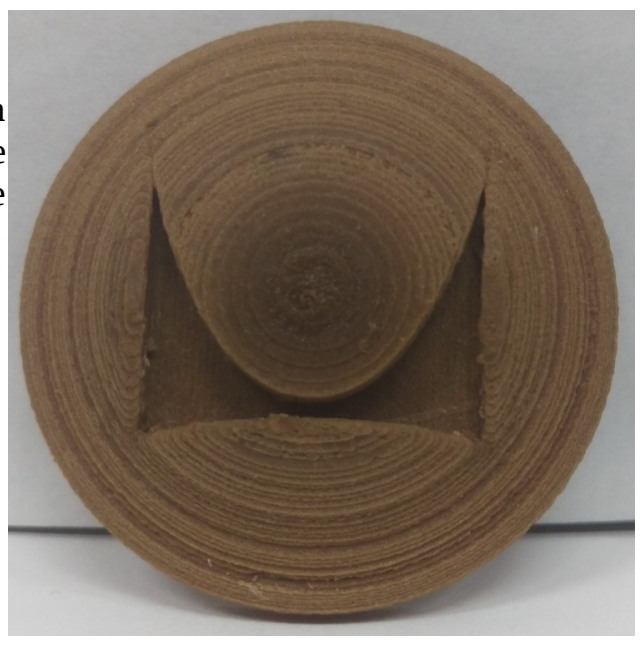

